\title{
Study on milling screw in algorithm of variable Positive Displacement Pump
}

\author{
CHEN Xin ${ }^{1,}$,, XU Mei Feng ${ }^{2, b}$ \\ ${ }^{1}$ Shenyang university of technology school of science, China \\ ${ }^{2}$ Shenyang university of technology school of science, China \\ a491079175@qq.com, b429071537@qq.com
}

Keywords: variable pump of volume; taper screw; similarity transformation; algorithm

\begin{abstract}
This thesis studies screw in algorithm of variable pump of volume based on normal screw, and puts forward algorithm of processing of taper screw, including the algorithm of data of a outline shape of arbitrary section, Segmentation of length of Segmentation algorithm, comparison algorithm of two outline shapes, and Verifies that the theory analysis is correct.
\end{abstract}

\section{Introduction}

Screw pump is one kind of positive displacement pump, with a single screw pump as an example. The single screw pump is a kind of internal meshing rotary displacement pump. When rotor is in the stator cavity to the eccentric to track the planetary rotation[1]. The interior of the pump flows slowly. The piston and the rotor motion both has low velocity and small wear; at the same time, we can get good self-priming performance by controlling the velocity and the structure parameters optimization design. The current research of screw pump is mostly cylindrical screw, inhalation chamber is the same as discharge chamber volume. If we change the conventional screw into a conical screw of variable displacement pump which the flow of inhalation is different from flow of excretion, it can be able to meet the different needs. In recent years, domestic volume pump made great process in the new structure, new materials and new technology and the variable. The research on volume pump is still in its initial stage, so research of the variable volume pump results will have certain value on theory and engineering significance [2]. This thesis studies the mathematical problems in the processing of variable Positive Displacement Pump, and puts forward solutions and laid a theoretical basis by prooing the feasible of method.

\section{Determine algorithm of tapered screw}

Algorithm of arbitrary cross-section profile.According to the end of data (such as big-endian), we can fit the section profile, by three times spine interpolation function [3]. Figure 1 is multi point-fitting of for two semi-crescent shape. Establishing coordinates of conical screw work piece is shown in Figure 2, and the taper screw radial length within the scope of any type of surface are the screw theory the similarity surface. So one section of tapered screw can be formed arbitrary surface profile by similarity transformation of plane coordinate.

The theory of similarity transformation of plane coordinate[4] is that the actual scale the hypothesis graph is the same in the direction of $X, Y$ and the characteristic is no changing the geometry of old mesh, the old network scaled registration to the new coordinates is by the whole translation, rotation. In the theory, the length of every point is equal to the new network. As shown in Figure 2, let $X O Y$ be the original rectangular coordinate system, and $x o^{\prime} y$ be the new rectangular coordinate system, the angle of two coordinate is $\alpha$, the translation distance of $o$ to $o^{\prime}$ is $A_{0}, B_{0}$ ant the actual scale in $X, Y$ is all $m$. 


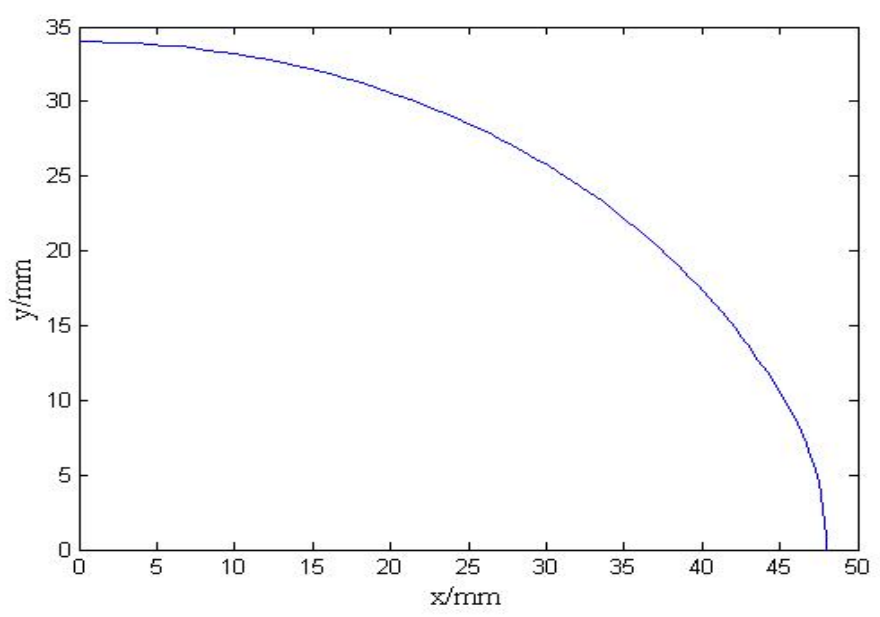

Figure 1 multi point-fitting of for two semi-crescent shape

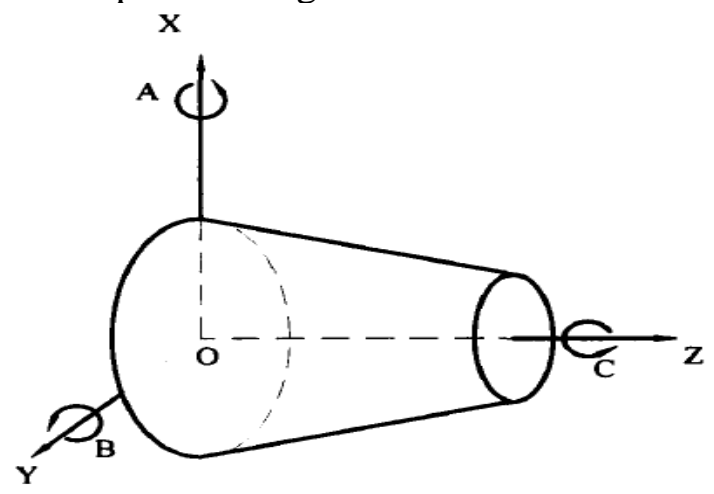

Figure2 rectangular coordinate system of porkpies

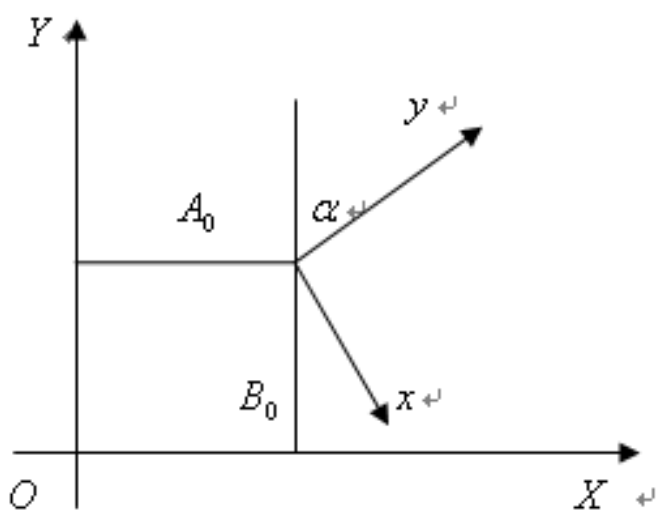

Figure3 plane coordinate similarity transformation

According to the principle of coordinate formula [5], its graphics is:

$\left\{\begin{array}{l}X=m(x \cos \alpha+y \sin \alpha)+A_{0} \\ Y=m(x \sin \alpha-y \cos \alpha)+B_{0}\end{array}\right.$

$A_{0} \quad, \quad B_{0} \quad$ is parameters of translation, $m$ is scale factor, $\alpha$ is rotation angle. $A_{1}=m \cos \alpha, B_{1}=m \sin \alpha$, then above equality changes to:

$\left\{\begin{array}{l}X=A_{0}+A_{1} x-B_{1} y \\ Y=B_{0}+B_{1} x-A_{1} y\end{array}\right.$

Let $X_{x}, Y_{y}$ be some known points, we have known that the actual coordinate of $X, Y$ subtracts the above result is: 


$$
\left\{\begin{array}{l}
Q_{x}=X-\left(A_{0}+A_{1} X-B_{1} y\right) \\
Q_{y}=Y-\left(B_{0}+B_{1} X-A_{1} y\right)
\end{array}\right.
$$

By the principle of least squares, with the $Q_{x}^{2}+Q_{y}^{2}$ be the minimum condition, as long as we know the 2 common point respectively in the 2 coordinate system of coordinates, we can obtained 4 transformation parameters (1). Due to the inevitable measurement error, we usually take 3 or more than 3 public points and list the error equation, and then we can calculate the transformation parameters. according to the least square method.

Algorithm of the length of the screw segmentation.Usually, in the process of cylindrical screw machining, the whole screw processing requires only one NC program. But for taper screw, if using a NC program, the program only can be placed on one end programming, to any other position even when the cutting process with appropriate cutter compensation, cutting to a certain position it will produce certain error. If the screw is longer, the error is larger. Therefore, the variable displacement pump taper screw processing the mutant alternative gradient processing method, the conical screw section. If the segmentation is more, reduces the screw processing efficiency, the overall number of independent NC system, increases the amount of storage, not suitable for practical use. So on the basis of the requirements for certain error, we must segment sections as little as possible, and each section of end for NC programming, in each segment by a mutation replaces the gradient, which ends in a phase error does not exceed the requirements of design.

Algorithm of two plane profile comparison.On the basis of given conical screw end profile data using three parameters spine function fitting out of the end surface profile. According to the plane coordinates similar transform theory, we can obtain the actual profile of conical screw, because of certain error in the coordinate transformation. It needs error analysis that the actual profile and after mutations are the theoretical profile.

\section{Examples of calculation}

The algorithm can obtain a variable volume pump taper screw processing procedures, and the procedures for a screw rotor is analyzed, desired parameters include: the length of the screw, screw head $\mathrm{z}=1000 \mathrm{~mm}$ number $\mathrm{n}=2$, tapered screw end data, small end data, axial feed rate $\mathrm{h}=20 \mathrm{~mm}$, accuracy of the $0.1 \mathrm{~mm}$, the calculated data as follows as shown in table 1 :

\begin{tabular}{lcl}
\hline Number & $\mathrm{x}$ & $\mathrm{y}$ \\
\hline 1 & 47.612352 & 0 \\
2 & 47.592514 & 0.902650 \\
3 & 47.532999 & 1.805301 \\
4 & 47.423887 & 2.698033 \\
5 & 47.285018 & 3.590764 \\
$\ldots$ & $\ldots$ & $\ldots$ \\
68 & 1.854897 & 33.695658 \\
69 & 0.962166 & 33.715497 \\
70 & 0.069434 & 33.725416 \\
\hline
\end{tabular}

(a) Data after similarity translation

\begin{tabular}{ccc}
\hline Number & xh & yh \\
\hline 1 & 47.612352 & 0 \\
2 & 47.592496 & 0.902650 \\
3 & 47.532632 & 1.805298 \\
4 & 47.423705 & 2.697981 \\
5 & 47.283465 & 3.590741 \\
$\ldots$ & $\ldots$ & $\ldots$ \\
68 & 0.961521 & 33.69290 \\
69 & 0.069388 & 33.702804 \\
70 & 0 & 33.702803 \\
\hline
\end{tabular}

(b) Date of cross section when $\mathrm{z}=20$

Table 1 date after calculating

The fitting curve obtained by two groups of data is shown in Figure 4 


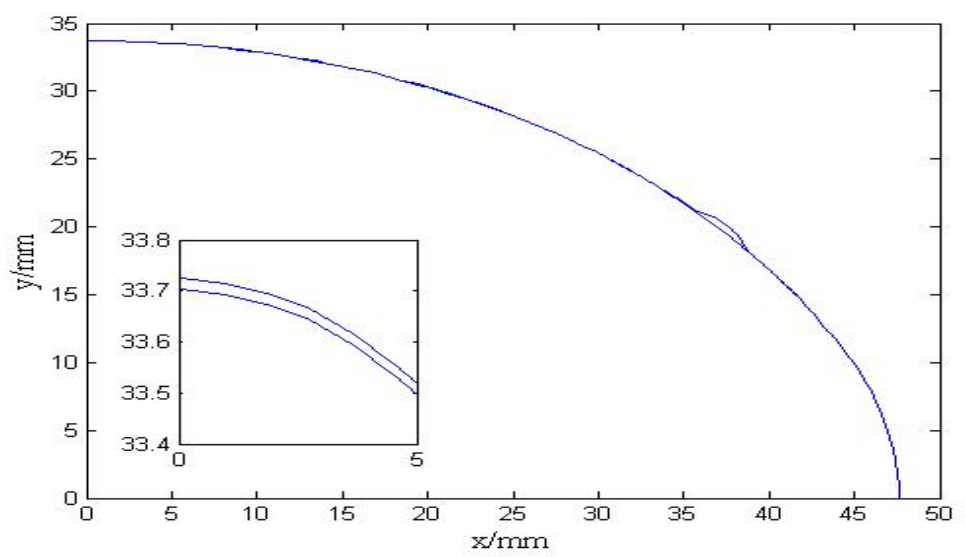

Figure 4 comparison of two fitting curve

According figure 4, the error between academic outline and actual outline can be meeting the requirements.

\section{Conclusions}

On the basis of the study on algorithm of variable volume pump screw processing, we get processing procedures by computer programming, and analyze the data of profile data and error, The results show that the algorithm in theory and in fact is feasible. In this paper, segmentation etc algorithms are used to determine variable volume pump screw working scheme, and according to the algorithm determine its processing program, based on the conventional screw are put forward on the basis of using similarity transformation, which can satisfy the actual need.

\section{References}

[1] Li Zaicheng, Li Zhibin. Single screw pump for transporting the medium consistency pulp [J]. Machinery and equipment,2007,10(28):28.

[2] Li Qiang, He Yurong. Research \& Innovation of volume pump products overview [J]. General Machinery,2002,11,12,38-40.

[3] WANG Ke, ZHAO Wei, GU Ke min, CHEN Xin, HAN Qi. Study on data approximate approach of spiral surface profile [J].journal of Shenyang university of technology,2002,4(2):91-94.

[4] Tian Fu Juan.The Development of Plane Coordinate Conversion Software [J].Pioneering with science \& technology monthly, 2006, 19(11):188-189.

[5] Liu Xin, Lu Fengnian, Shang Xueli. Design and Implementation of the Plane Similar to Transform Software [J]. Computer \& Digital Engineering,2009,11(37):30-32. 